\title{
A FAMILY OF INCOMPLETE HURWITZ-LERCH ZETA FUNCTIONS OF TWO VARIABLES
}

\author{
H. M. SRIVASTAVA, RECEP ŞAHIN, AND OĞUZ YAĞCI \\ Received 06 October, 2019
}

\begin{abstract}
Inspired essentially by the work [H. M. Srivastava, M. A. Chaudhry and R. P. Agarwal [The incomplete Pochhammer symbols and their applications to hypergeometric and related functions, Integral Transforms Spec. Funct. 23 (2012), 659-683] (see [16])], we introduce the families of the incomplete Hurwitz-Lerch Zeta functions of two variables. We then give the integral representations including the Mellin-Barnes contour integral representation, summation formulas, derivative formulas and recurrence relations for the incomplete Hurwitz-Lerch Zeta functions of two variables.
\end{abstract}

2010 Mathematics Subject Classification: 11M06; 11M35; 33B15; 33C60; 11M68; 33C65; 33C90

Keywords: Gamma function, Incomplete gamma functions, Beta function, Pochhammer symbol, Incomplete Pochhammer symbols, Hurwitz-Lerch Zeta function, Hurwitz-Lerch Zeta function of two variables, Incomplete confluent hypergeometric functions, Appell hypergeometric function, Incomplete Appell hypergeometric functions, Humbert hypergeometric functions of two variables, Integral representations, Mellin-Barnes integral formula, Summation formula, Derivative formulas, Recurrence relation

\section{INTRODUCTION, DEFINITIONS AND PRELIMINARIES}

The classical incomplete Gamma functions $\gamma(s, x)$ and $\Gamma(s, x)$ satisfy the following decomposition formula $[3,4,7,16]$ :

$$
\gamma(s, x)+\Gamma(s, x)=\Gamma(s)=\int_{0}^{\infty} t^{s-1} \exp (-t) \mathrm{d} t \quad(\Re(s)>0) .
$$

By using each of these classical incomplete Gamma functions $\gamma(s, x)$ and $\Gamma(s, x)$, the incomplete Pochhammer symbols $(\lambda, x)_{v}$ and $[\lambda, x]_{v}$ were defined, for $x \geqq 0$ and $\lambda, \nu \in$ $\mathbb{C}$, by Srivastava et al. (see, for details, [16]). In fact, these incomplete Pochhammer symbols $(\lambda, x)_{v}$ and $[\lambda, x]_{v}$ satisfy the decomposition formula:

$$
(\lambda, x)_{v}+[\lambda, x]_{v}=(\lambda)_{v}:=\frac{\Gamma(\lambda+v}{\Gamma(\lambda)}=\frac{1}{\Gamma(\lambda)} \int_{0}^{\infty} t^{\lambda+v-1} \exp (-t) \mathrm{d} t
$$

provided that the Gamma quotient as well as the infinite integral exist. Furthermore, Srivastava et al. [16] investigated many properties of the generalized incomplete 
hypergeometric functions by the help of these representations (see also a closelyrelated recent work [23] dealing mainly with such much more general functions as the incomplete $H$-functions and the incomplete $\bar{H}$-functions).

Many researchers studied many different generalizations and extensions of the familiar Hurwitz-Lerch Zeta function $\Phi(z, r, \alpha)$ by inserting certain additional parameters to the series representation of the Hurwitz-Lerch Zeta function. The interested readers can refer to these earlier publications for further researches and applications [5,8,10-15, 17-19,21, 24]. Recently, Choi and Parmar [6] introduced and studied the following extension of the generalized Hurwitz-Lerch Zeta function in two variables:

$$
\begin{gathered}
\Phi_{a, b, b^{\prime} ; c}(z, t, r, \alpha)=\sum_{k, l=0}^{\infty} \frac{(a)_{k+l}(b)_{k}\left(b^{\prime}\right)_{l}}{(c)_{k+l} k ! l !} \frac{z^{k} t^{l}}{(k+l+\alpha)^{r}} \\
\left(a, b, b^{\prime} \in \mathbb{C} ; c, \alpha \in \mathbb{C} \backslash \mathbb{Z}_{0} ; r, z, t \in \mathbb{C} \text { when }|z|<1 \text { and }|t|<1 ;\right. \\
\left.\Re\left(r-c-a-b-b^{\prime}\right)>1 \text { when }|z|=1 \text { and }|t|=1\right) .
\end{gathered}
$$

Motivated by the above-mentioned works, our aim here is to investigate an extended family of the generalized incomplete Hurwitz-Lerch Zeta functions of two variables. In particular, we obtain integral representations including the Mellin-Barnes contour integral representation, derivative formulas, summation formulas, series relations and recurrence relations. We choose to record some of our results for only one member of the above extended family of the generalized incomplete HurwitzLerch Zeta functions of two variables. Analogous results can be derived fairly easily for the other member of the above extended family of the generalized incomplete Hurwitz-Lerch Zeta functions of two variables.

\section{THE INCOMPLETE EXTENDED HURWITZ-LERCH ZETA FUNCTIONS OF TWO VARIABLES}

In this section, we introduce the following family of the incomplete extended Hurwitz-Lerch Zeta functions of two variables:

$$
\phi_{a, b, b^{\prime} ; c}(z, t, r, \alpha)=\sum_{k, l=0}^{\infty} \frac{(a, x)_{k+l}(b)_{k}\left(b^{\prime}\right)_{l}}{(c)_{k+l} k ! l !} \frac{z^{k} t^{l}}{(k+l+\alpha)^{r}}
$$

and

$$
\begin{gathered}
\varphi_{a, b, b^{\prime} ; c}(z, t, r, \alpha)=\sum_{k, l=0}^{\infty} \frac{[a, x]_{k+l}(b)_{k}\left(b^{\prime}\right)_{l}}{(c)_{k+l} k ! l !} \frac{z^{k} t^{l}}{(k+l+\alpha)^{r}} \\
\left(x \geqq 0 ; a, b, b^{\prime} \in \mathbb{C} ; c, \alpha \in \mathbb{C} \backslash \mathbb{Z}_{0} ; r, z, t \in \mathbb{C} \text { when }|z|<1 \text { and }|t|<1 ;\right.
\end{gathered}
$$




$$
\left.\mathfrak{R}\left(r-c-a-b-b^{\prime}\right)>1 \text { when }|z|=1 \text { and }|t|=1\right) .
$$

By virtue of (1.2), these families of the incomplete extended Hurwitz-Lerch Zeta functions of two variables satisfy a decomposition formula in terms of $\Phi_{a, b, b^{\prime} ; c}(z, t, r, \alpha)$, which is the extension of the generalized Hurwitz-Lerch function of two variables in [6].

Remark 1. The limit cases of the families of the incomplete Hurwitz-Lerch Zeta functions of two variables are given by

$$
\begin{aligned}
\phi_{a, b ; c}^{*}(z, t, r, \alpha) & =\lim _{b^{\prime} \rightarrow \infty}\left\{\phi_{a, b, b^{\prime} ; c}\left(z, \frac{t}{b^{\prime}}, r, \alpha\right)\right\} \\
& =\sum_{k, l=0}^{\infty} \frac{(a, x)_{k+l}(b)_{k}}{(c)_{k+l} k ! l !} \frac{z^{k} t^{l}}{(k+l+\alpha)^{r}}
\end{aligned}
$$

and

$$
\begin{gathered}
\varphi_{a, b ; c}^{*}(z, t, r, \alpha)=\lim _{b^{\prime} \rightarrow \infty}\left\{\varphi_{a, b, b^{\prime} ; c}\left(z, \frac{t}{b^{\prime}}, r, \alpha\right)\right\} \\
=\sum_{k, l=0}^{\infty} \frac{[a, x]_{k+l}(b)_{k}}{(c)_{k+l} k ! l !} \frac{z^{k} t^{l}}{(k+l+\alpha)^{r}} \\
\left(x \geqq 0 ; a, b, b^{\prime} \in \mathbb{C} ; c, \alpha \in \mathbb{C} \backslash \mathbb{Z}_{0} ; r, z, t \in \mathbb{C} \text { when }|z|<1 \text { and }|t|<1 ;\right. \\
\left.\Re\left(r-c-a-b-b^{\prime}\right)>1 \text { when }|z|=1 \text { and }|t|=1\right) .
\end{gathered}
$$

\section{INTEGRAL REPRESENTATIONS}

For the parameters $a, b, b^{\prime} \in \mathbb{C} ; c \in \mathbb{C} \backslash \mathbb{Z}_{0}$, we make use of the following incomplete first Appell hypergeometric functions (see [7]):

$$
\gamma_{1}\left[(a, x), b, b^{\prime} ; c ; x, y\right]=\sum_{k, l=0}^{\infty} \frac{(a, x)_{k+l}(b)_{k}\left(b^{\prime}\right)_{l}}{(c)_{k+l}} \frac{x^{k} y^{l}}{k ! l !}
$$

and

$$
\Gamma_{1}\left[(a, x), b, b^{\prime} ; c ; x, y\right]=\sum_{k, l=0}^{\infty} \frac{[a, x]_{k+l}(b)_{k}\left(b^{\prime}\right)_{l}}{(c)_{k+l}} \frac{x^{k} y^{l}}{k ! l !},
$$

which satisfy the following decomposition formula:

$$
\gamma_{1}\left[(a, x), b, b^{\prime} ; c ; x, y\right]+\Gamma_{1}\left[(a, x), b, b^{\prime} ; c ; x, y\right]=F_{1}\left(a, b, b^{\prime} ; c ; x, y\right)
$$

where $F_{1}\left(a, b, b^{\prime} ; c ; x, y\right)$ is the first Appell hypergeometric function (see, for example, $[1,2,9,20,22])$. 
Theorem 1. The following integral representation for the function $\varphi_{a, b, b^{\prime} ; c}(z, t, r, \alpha)$ defined by (2.2) holds true:

$$
\begin{array}{r}
\varphi_{a, b, b^{\prime} ; c}(z, t, r, \alpha) \\
=\frac{1}{\Gamma(r)} \int_{0}^{\infty} u^{r-1} \exp (-\alpha u) \Gamma_{1}\left[(a, x), b, b^{\prime} ; c ; z e^{-u}, t e^{-u}\right] \mathrm{d} u \\
(x \geqq 0 ; \min \{\Re(r), \Re(\alpha)\}>0 \text { when }|z| \leqq 1 \quad(z \neq 1) \\
\text { and }|t| \leqq 1(t \neq 1) ; \Re(r)>0 \text { when } z=t=1) .
\end{array}
$$

Proof. Applying the following Eulerian integral (see [6]):

$$
\begin{gathered}
\frac{1}{(k+l+r)^{\alpha}}=\frac{1}{\Gamma(r)} \int_{0}^{\infty} u^{r-1} \exp (-\alpha u-k u-l u) \mathrm{d} u \\
\left(\min \{\Re(r), \Re(\alpha)\}>0 ; k, l \in \mathbb{N}_{0}\right)
\end{gathered}
$$

to the equation (2.2) and inverting the order of summation and integration, followed by using (3.2), we can get the desired result (3.4).

Theorem 2. The following integral representations for the function $\varphi_{a, b, b^{\prime} ; c}(z, t, r, \alpha)$ defined by (2.2) hold true:

$$
\begin{aligned}
\varphi_{a, b, b^{\prime} ; c}(z, t, r, \alpha)= & \frac{1}{\Gamma(r) \Gamma(a)} \int_{x}^{\infty} \int_{0}^{\infty} u^{r-1} v_{1}^{a-1} \exp \left(-\alpha u-v_{1}\right) \\
& \cdot \Phi_{2}\left[b, b^{\prime} ; c ; z e^{-u} v_{1}, t e^{-u} v_{1}\right] \mathrm{d} u \mathrm{~d} v_{1}
\end{aligned}
$$

$$
\begin{gathered}
\varphi_{a, b, b^{\prime} ; c}(z, t, r, \alpha) \\
=\frac{1}{\Gamma(r) \Gamma(b) \Gamma\left(b^{\prime}\right)} \int_{0}^{\infty} \int_{0}^{\infty} \int_{0}^{\infty} u^{r-1} v_{2}^{b-1} v_{3}^{b^{\prime}-1} \exp \left(-\alpha u-v_{2}-v_{3}\right) \\
\cdot{ }_{1} \Gamma_{1}\left[(a, x) ; c ; z e^{-u} v_{2}+t e^{-u} v_{3}\right] \mathrm{d} u \mathrm{~d} v_{2} \mathrm{~d} v_{3}, \\
\varphi_{a, b, b^{\prime} ; c}(z, t, r, \alpha)=\frac{1}{\Gamma(r) \Gamma(a) \Gamma(b) \Gamma\left(b^{\prime}\right)} \\
\cdot \int_{0}^{\infty} \int_{0}^{\infty} \int_{x}^{\infty} \int_{0}^{\infty} u^{r-1} v_{1}^{a-1} v_{2}^{b-1} v_{3}^{b^{\prime}-1} \exp \left(-\alpha u-v_{1}-v_{2}-v_{3}\right) \\
\cdot{ }_{0} F_{1}\left[-; c ; z e^{-u} v_{1} v_{2}+t e^{-u} v_{1} v_{3}\right] \mathrm{d} u \mathrm{~d} v_{1} \mathrm{~d} v_{2} \mathrm{~d} v_{3}, \\
\varphi_{a, b, b^{\prime} ; c+1}(-z,-t, r, \alpha)=\frac{\Gamma(c+1)}{\Gamma(r) \Gamma(a) \Gamma(b) \Gamma\left(b^{\prime}\right)} \\
\cdot \int_{0}^{\infty} \int_{0}^{\infty} \int_{x}^{\infty} \int_{0}^{\infty} u^{r-1} v_{1}^{a-\frac{c}{2}-1} v_{2}^{b-1} v_{3}^{b^{\prime}-1}
\end{gathered}
$$




$$
\begin{aligned}
& \cdot \exp \left(-\alpha u-v_{1}-v_{2}-v_{3}\right)\left(z e^{-u} v_{2}+t e^{-u} v_{3}\right)^{-\frac{c}{2}} \\
& \cdot J_{c}\left(2 \sqrt{z e^{-u} v_{1} v_{2}+t e^{-u} v_{1} v_{3}}\right) \mathrm{d} u \mathrm{~d} v_{1} \mathrm{~d} v_{2} \mathrm{~d} v_{3}
\end{aligned}
$$

and

$$
\begin{gathered}
\varphi_{a, b, b^{\prime} ; c+1}(z, t, r, \alpha)=\frac{\Gamma(c+1)}{\Gamma(r) \Gamma(a) \Gamma(b) \Gamma\left(b^{\prime}\right)} \\
\cdot \int_{0}^{\infty} \int_{0}^{\infty} \int_{x}^{\infty} \int_{0}^{\infty} u^{r-1} v_{1}^{a-\frac{c}{2}-1} v_{2}^{b-1} v_{3}^{b^{\prime}-1} \\
\cdot \exp \left(-\alpha u-v_{1}-v_{2}-v_{3}\right)\left(z e^{-u} v_{2}+t e^{-u} v_{3}\right)^{-\frac{c}{2}} \\
\cdot I_{c}\left(2 \sqrt{z e^{-u} v_{1} v_{2}+t e^{-u} v_{1} v_{3}}\right) \mathrm{d} u \mathrm{~d} v_{1} \mathrm{~d} v_{2} \mathrm{~d} v_{3} \\
(x \geqq 0 ; \min \{\Re(r), \Re(\alpha)\}>0 ; \max \{\Re(z), \Re(t)\}<1 ; \Re(a)>0 \text { when } x=0),
\end{gathered}
$$

provided that conditions analogous to those that are listed above with (3.10), are satisfied also for all the assertions (3.6) to (3.9), $J_{v}(z)$ and $I_{v}(z)$ being the Bessel and the modified Bessel functions, respectively.

Proof. If we use following integral representation of the incomplete first Appell hypergeometric function $\Gamma_{1}$ in (3.4) and interchange the order of summation and integration, we can obtain the desired result (3.6):

$$
\Gamma_{1}\left[(a, x), b, b^{\prime} ; c ; z, t\right]=\frac{1}{\Gamma(a)} \int_{x}^{\infty} v_{1}^{a-1} \exp \left(-v_{1}\right) \Phi_{2}\left[b, b^{\prime} ; c ; z v_{1}, t v_{1}\right] \mathrm{d} v_{1},
$$

where, for convergence, $\Re(a)>0$.

In the same way as above, by using the other integral representations of the incomplete first Appell hypergeometric function $\Gamma_{1}$ in [7], we can obtain the equations (3.7) to (3.10).

Theorem 3. The following integral representations for the function $\varphi_{a, b, b^{\prime} ; c}(z, t, r, \alpha)$ defined by (2.2) hold true:

$$
\begin{gathered}
\varphi_{a, b, b^{\prime} ; c}(z, t, r, \alpha)=\frac{\Gamma(c)}{\Gamma(r) \Gamma(a) \Gamma(b) \Gamma\left(b^{\prime}\right) \Gamma\left(c-b-b^{\prime}\right)} \\
\cdot \int_{0}^{1} \int_{0}^{1} \int_{x}^{\infty} \int_{0}^{\infty} u^{r-1} v_{1}^{a-1} \mu^{b-1} \sigma^{b^{\prime}-1} \\
\cdot \exp \left(-\alpha u-v_{1}+z e^{-u} v_{1} \mu+t e^{-u} v_{1}(1-\mu) \sigma\right) \\
\cdot(1-\mu)^{c-b-1}(1-\sigma)^{c-b-b^{\prime}-1} \mathrm{~d} u \mathrm{~d} v_{1} \mathrm{~d} \mu \mathrm{d} \sigma, \\
\varphi_{a, b, b^{\prime} ; c}(z, t, r, \alpha)=\frac{\Gamma(\delta)}{\Gamma(r) \Gamma(a) \Gamma(b) \Gamma\left(b^{\prime}\right) \Gamma\left(\delta-b-b^{\prime}\right)}
\end{gathered}
$$




$$
\begin{aligned}
& \int_{0}^{1} \int_{0}^{1} \int_{x}^{\infty} \int_{0}^{\infty} u^{r-1} v_{1}^{a-1} \mu^{b-1} \sigma^{b^{\prime}-1} \\
& \cdot \exp \left(-\alpha u-v_{1}+z e^{-u} v_{1} \mu+t e^{-u} v_{1}(1-\mu) \sigma\right) \\
& \cdot{ }_{1} F_{1}\left[c-\delta ; c ;-z e^{-u} v_{1} \mu-t e^{-u} v_{1}(1-\mu) \sigma\right] \\
& \cdot(1-\mu)^{\delta-b-1}(1-\sigma)^{\delta-b-b^{\prime}-1} \mathrm{~d} u \mathrm{~d} v_{1} \mathrm{~d} \mu \mathrm{d} \sigma, \\
& \varphi_{a, b, b^{\prime} ; c}(z, t, r, \alpha)=\frac{\Gamma(c)}{\Gamma(r) \Gamma(a) \Gamma\left(\delta_{1}\right) \Gamma\left(b^{\prime}\right) \Gamma\left(c-\delta_{1}-b^{\prime}\right)} \\
& \cdot \int_{0}^{1} \int_{0}^{1} \int_{x}^{\infty} \int_{0}^{\infty} u^{r-1} v_{1}^{a-1} \mu^{\delta_{1}-1} \sigma^{b^{\prime}-1} \\
& \cdot \exp \left(-\alpha u-v_{1}+z e^{-u} v_{1} \mu+t e^{-u} v_{1}(1-\mu) \sigma\right) \\
& \text {. }{ }_{1} F_{1}\left[\delta_{1}-b ; \delta_{1} ;-z e^{-u} v_{1} \mu\right] \\
& \cdot(1-\mu)^{c-\delta_{1}-1}(1-\sigma)^{c-\delta_{1}-b^{\prime}-1} \mathrm{~d} u \mathrm{~d} v_{1} \mathrm{~d} \mu \mathrm{d} \sigma, \\
& \varphi_{a, b, b^{\prime} ; c}(z, t, r, \alpha)=\frac{\Gamma(c)}{\Gamma(r) \Gamma(a) \Gamma\left(\delta_{1}\right) \Gamma\left(b^{\prime}\right) \Gamma\left(c-\delta_{1}-b^{\prime}\right)} \\
& \cdot \int_{0}^{1} \int_{0}^{1} \int_{x}^{\infty} \int_{0}^{\infty} u^{r-1} v_{1}^{a-1} \mu^{\delta_{1}-1} \sigma^{b^{\prime}-1} \\
& \cdot \exp \left(-\alpha u-v_{1}+z e^{-u} v_{1} \mu+t e^{-u} v_{1}(1-\mu)\right) \\
& \text {. }{ }_{1} F_{1}\left[b-\delta_{1} ; c-\delta_{1}-b^{\prime} ; z e^{-u} v_{1}(1-\mu)(1-\sigma)\right] \\
& \cdot(1-\mu)^{c-\delta_{1}-1}(1-\sigma)^{c-\delta_{1}-b^{\prime}-1} \mathrm{~d} u \mathrm{~d} v_{1} \mathrm{~d} \mu \mathrm{d} \sigma, \\
& \varphi_{a, b, b^{\prime} ; c}(z, t, r, \alpha)=\frac{\Gamma(c)}{\Gamma(r) \Gamma(a) \Gamma\left(\delta_{1}\right) \Gamma\left(\delta_{2}\right) \Gamma\left(c-\delta_{1}-\delta_{2}\right)} \\
& \cdot \int_{0}^{1} \int_{0}^{1} \int_{x}^{\infty} \int_{0}^{\infty} u^{r-1} v_{1}^{a-1} \mu^{\delta_{1}-1} \sigma^{\delta_{2}-1} \\
& \cdot \exp \left(-\alpha u-v_{1}+z e^{-u} v_{1} \mu+t e^{-u} v_{1}(1-\mu) \sigma\right) \\
& \text {. }{ }_{1} F_{1}\left[\delta_{1}-b ; \delta_{1} ;-z e^{-u} v_{1} \mu\right]{ }_{1} F_{1}\left[\delta_{2}-b^{\prime} ; \delta_{2} ;-t e^{-u} v_{1}(1-\mu) \sigma\right] \\
& \cdot(1-\mu)^{c-\delta_{1}-1}(1-\sigma)^{c-\delta_{1}-\delta_{2}-1} \mathrm{~d} u \mathrm{~d} v_{1} \mathrm{~d} \mu \mathrm{d} \sigma
\end{aligned}
$$

and

$$
\begin{gathered}
\varphi_{a, b, b^{\prime} ; c}(z, t, r, \alpha)=\frac{\Gamma(c)}{\Gamma(r) \Gamma(a) \Gamma\left(\delta_{1}\right) \Gamma\left(\delta_{2}\right) \Gamma\left(c-\delta_{1}-\delta_{2}\right)} \\
\cdot \int_{0}^{1} \int_{0}^{1} \int_{x}^{\infty} \int_{0}^{\infty} u^{r-1} v_{1}^{a-1} \mu^{\delta_{1}-1} \sigma^{\delta_{2}-1} \\
\cdot \exp \left(-\alpha u-v_{1}+z e^{-u} v_{1} \mu+t e^{-u} v_{1}(1-\mu) \sigma\right)
\end{gathered}
$$




$$
\begin{aligned}
& \cdot \Phi_{2}\left[b-\delta_{1}, b^{\prime}-\delta_{2} ; c ;-z e^{-u} v_{1}(1-\mu)(1-\sigma), t e^{-u} v_{1}(1-\mu)(1-\sigma)\right] \\
& \cdot(1-\mu)^{c-\delta_{1}-1}(1-\sigma)^{c-\delta_{1}-\delta_{2}-1} \mathrm{~d} u \mathrm{~d} v_{1} \mathrm{~d} \mu \mathrm{d} \sigma
\end{aligned}
$$

provided that the second member of each of the assertions (3.11) to (3.16) exists.

Proof. We recall from [2] that

$$
\begin{gathered}
\Phi_{2}\left[b, b^{\prime} ; c ; z, t\right]=\frac{\Gamma(c)}{\Gamma(r) \Gamma(a) \Gamma(b) \Gamma\left(b^{\prime}\right) \Gamma\left(c-b-b^{\prime}\right)} \\
\cdot \int_{0}^{1} \int_{0}^{1} \mu^{b-1} \sigma^{b^{\prime}-1} \exp (z \mu+t(1-\mu) \sigma) \\
\cdot(1-\mu)^{c-b-1}(1-\sigma)^{c-b-b^{\prime}-1} \mathrm{~d} \mu \mathrm{d} \sigma .
\end{gathered}
$$

If we use (3.17) in (3.6) and interchange order of summation and integration, we can get the desired result (3.11).

In the same way as above, by using the other integral representations of the Humbert function of two variables $\Phi_{2}$ (see [2]), we can obtain the equations (3.12) to (3.16).

\section{MELLin-BARnes CONTOUR INTEGRAL REPRESENTATION}

In this section, we give the Mellin-Barnes contour integral representation for the families of the incomplete Hurwitz-Lerch functions defined by (2.1) and (2.2).

Theorem 4. The Mellin-Barnes contour integral representation for (2.2) is given by

$$
\begin{aligned}
\varphi_{a, b, b^{\prime} ; c}(z, t, r, \alpha)=\frac{\Gamma(c)}{\Gamma(a) \Gamma(b) \Gamma\left(b^{\prime}\right)} \frac{1}{2 \pi i} \int_{\mathcal{L}_{z}} \int_{\mathcal{L}_{t}} \\
\cdot \frac{\Gamma(-\mu) \Gamma(-v) \Gamma(a+\mu+v, x) \Gamma(b+\mu) \Gamma\left(b^{\prime}+v\right)[\Gamma(\mu+v+\alpha)]^{r}}{\Gamma(c+\mu+v)[\Gamma(\mu+v+\alpha+1)]^{r}} \\
\cdot(-z)^{\mu}(-t)^{v} \mathrm{~d} \mu \mathrm{d} v \quad(|\arg (-z)|<\pi ;|\arg (-t)|<\pi),
\end{aligned}
$$

where it is assumed that the poles of the integrand in (4.1) are simple and the MellinBernes type contours $\mathcal{L}_{z}$ and $\mathcal{L}_{t}$ of integration are so chosen that the poles of $\Gamma(-\mu)$ and $\Gamma(-v)$ can be separated from those of $\Gamma(b+\mu)$ and $\Gamma\left(b^{\prime}+v\right)$ with indentations, if needed.

Proof. Taking the sum of the residues at the poles of $\Gamma(-\mu)$ at the points $\mu=k$ $\left(k \in \mathbb{N}_{0}\right)$ and the poles of $\Gamma(-v)$ at the points $v=l\left(l \in \mathbb{N}_{0}\right)$ in the equation (4.1), we readily get the following series expansion:

$$
\varphi_{a, b, b^{\prime} ; c}(z, t, r, \alpha)=\frac{\Gamma(c)}{\Gamma(a) \Gamma(b) \Gamma\left(b^{\prime}\right)}
$$




$$
\cdot \sum_{k, l=0}^{\infty} \frac{\Gamma(a+k+l, x) \Gamma(b+k) \Gamma\left(b^{\prime}+l\right)}{\Gamma(c+k+l) k ! l !} \frac{z^{k} t^{l}}{(k+l+\alpha)^{r}},
$$

which completes the proof of Theorem 4 .

\section{A SUMMATION FORMULA}

Theorem 5. A summation formula for the function $\varphi_{a, b, b^{\prime} ; c}(z, t, r, \alpha)$ defined by (2.2) is given, for $|\beta|<\alpha$ and $r \neq 1$, by

$$
\varphi_{a, b, b^{\prime} ; c}(z, t, r, \alpha-\beta)=\sum_{p=0}^{\infty} \frac{(r)_{p}}{p !} \varphi_{a, b, b^{\prime} ; c}(z, t, r+p, \alpha) \beta^{p} .
$$

Proof. Using the definition (2.2) in the left-hand side of the assertion (5.1), we find that

$$
\begin{aligned}
\varphi_{a, b, b^{\prime} ; c}(z, t, r, \alpha-\beta) \\
=\sum_{k, l=0}^{\infty} \frac{[a, x]_{k+l}(b)_{k}\left(b^{\prime}\right)_{l}}{(c)_{k+l} k ! l !} \frac{z^{k} t^{l}}{(k+l+\alpha)^{r}}\left(1-\frac{\beta}{k+l+\alpha}\right)^{-r} \\
=\sum_{k, l=0}^{\infty} \frac{[a, x]_{k+l}(b)_{k}\left(b^{\prime}\right)_{l}}{(c)_{k+l} k ! l !} \frac{z^{k} t^{l}}{(k+l+\alpha)^{r}}\left(\sum_{p=0}^{\infty} \frac{(r)_{p}}{p !} \frac{\beta^{p}}{(k+l+\alpha)^{p}}\right) \\
=\sum_{p=0}^{\infty} \frac{(r)_{p}}{p !}\left(\sum_{k, l=0}^{\infty} \frac{[a, x]_{k+l}(b)_{k}\left(b^{\prime}\right)_{l}}{(c)_{k+l} k ! l !} \frac{z^{k} t^{l}}{(k+l+\alpha)^{r+p}}\right) \beta^{p},
\end{aligned}
$$

which readily gives the desired result (5.1).

\section{Derivative Formulas}

In this section, we consider the derivative formulas for the families of the incomplete Hurwitz-Lerch functions defined by (2.1) and (2.2). In view of the abovementioned decomposition formula resulting from (2.1) and (2.2), we choose to record here the derivative formulas for the function $\varphi_{a, b, b^{\prime} ; c}(z, t, r, \alpha)$ defined by (2.2). The corresponding results for the function $\phi_{a, b, b^{\prime} ; c}(z, t, r, \alpha)$ defined by (2.1) can be derived fairly easily.

Theorem 6. Each of the following derivative formulas for the function $\varphi_{a, b, b^{\prime} ; c}(z, t, r, \alpha)$ defined by (2.2) holds true:

$$
\begin{gathered}
\frac{\partial^{k}}{\partial z^{k}}\left\{\varphi_{a, b, b^{\prime} ; c}(z, t, r, \alpha)\right\}=\frac{(a)_{k}(b)_{k}}{(c)_{k}} \\
\cdot \varphi_{a+k, b+k, b^{\prime} ; c+k}(z, t, r, \alpha+k) \quad\left(k \in \mathbb{N}_{0}\right), \\
\frac{\partial^{l}}{\partial t^{l}}\left\{\varphi_{a, b, b^{\prime} ; c}(z, t, r, \alpha)\right\}=\frac{(a)_{l}\left(b^{\prime}\right)_{l}}{(c)_{l}}
\end{gathered}
$$




$$
\cdot \varphi_{a+l, b, b^{\prime}+l ; c+l}(z, t, r, \alpha+l) \quad\left(l \in \mathbb{N}_{0}\right)
$$

and

$$
\begin{aligned}
& \frac{\partial^{k+l}}{\partial z^{k} \partial t^{l}}\left\{\varphi_{a, b, b^{\prime} ; c}(z, t, r, \alpha)\right\}=\frac{(a)_{k+l}(b)_{k}\left(b^{\prime}\right)_{l}}{(c)_{k+l}} \\
& \cdot \varphi_{a+k+l, b+k, b^{\prime}+l ; c+k+l}(z, t, r, \alpha+k+l) \quad\left(k, l \in \mathbb{N}_{0}\right) .
\end{aligned}
$$

Proof. Differentiating both sides of the series definition (2.2) with respect to the variable $z$, which is valid under the conditions in (2.2), we obtain

$$
\frac{\partial}{\partial z}\left\{\varphi_{a, b, b^{\prime} ; c}(z, t, r, \alpha)\right\}=\sum_{l=0}^{\infty} \sum_{k=1}^{\infty} \frac{[a, x]_{k+l}(b)_{k}\left(b^{\prime}\right)_{l}}{(c)_{k+l}(k-1) ! l !} \frac{z^{k-1} t^{l}}{(k+l+\alpha)^{r}} .
$$

Putting $k-1=k^{\prime}$ in (6.4) and dropping the prime on $k$, we get

$$
\begin{gathered}
\frac{\partial}{\partial z}\left\{\varphi_{a, b, b^{\prime} ; c}(z, t, r, \alpha)\right\}=\sum_{l=0}^{\infty} \sum_{k=0}^{\infty} \frac{[a, x]_{k+l+1}(b)_{k+1}\left(b^{\prime}\right)_{l}}{(c)_{k+l+1} k ! l !} \\
\cdot \frac{z^{k} t^{l}}{(k+l+1+\alpha)^{r}} .
\end{gathered}
$$

Next, by applying the following identities:

$$
[\lambda, x]_{k+1}=\lambda[\lambda+1, x]_{k} \quad \text { and } \quad(\lambda)_{k+1}=\lambda(\lambda+1)_{k} \quad\left(k \in \mathbb{N}_{0}\right)
$$

in the last equation (6.5), we have

$$
\frac{\partial}{\partial z}\left\{\varphi_{a, b, b^{\prime} ; c}(z, t, r, \alpha)\right\}=\frac{a b}{c} \varphi_{a+1, b+1, b^{\prime} ; c+1}(z, t, r, \alpha+1) .
$$

Finally, if we successively differentiate the right-hand side of (6.6) $k-1$ times with respect to the variable $z$, we have the assertion (6.1) of Theorem 6 .

In a similar manner, we can derive the derivative formulas (6.2) and (6.3) asserted by Theorem 6 .

Theorem 7. The derivative formulas for the function $\varphi_{a, b, b^{\prime} ; c}(z, t, r, \alpha)$ defined by (2.2) are given by

$$
\begin{gathered}
D_{t}^{n}\left\{t^{b^{\prime}+n-1} \varphi_{a, b, b^{\prime} ; c}(z, t, r, \alpha)\right\}=t^{b-1}\left(b^{\prime}\right)_{n} \varphi_{a, b, b^{\prime}+n ; c}(z, t, r, \alpha), \\
D_{t}^{n}\left\{t^{c-1} \varphi_{a, b, b^{\prime} ; c}(z t, t, r, \alpha)\right\} \\
=(-1)^{n} t^{c-n-1}(1-c)_{n} \varphi_{a, b, b^{\prime} ; c-n}(z t, t, r, \alpha)
\end{gathered}
$$

and

$$
\begin{gathered}
D_{t}^{n}\left\{t^{c-b-1} \varphi_{a, b, b^{\prime} ; c}(z, t, r, \alpha)\right\}=(-1)^{n} t^{c-b-n-1}(b-c+1)_{n} \\
\cdot \sum_{p=0}^{n}\left(\begin{array}{l}
n \\
p
\end{array}\right) \frac{(a)_{p}\left(b^{\prime}\right)_{p} t^{p}}{(c)_{p}(c-b-n)_{p}} \varphi_{a+p, b, b^{\prime}+p ; c+p}(z, t, r, \alpha)
\end{gathered}
$$


where

$$
D_{t}^{n}\{f(z, t)\}=\frac{\partial^{n}}{\partial t^{n}}\{f(z, t)\}
$$

Proof. Using the definition (2.2) in the left-hand side of the assertion (6.7), we have

$$
\begin{aligned}
D_{t}^{n}\left\{t^{b^{\prime}+}\right. & \left.=\varphi_{a, b, b^{\prime} ; c}(z, t, r, \alpha)\right\} \\
& =D_{t}^{n}\left\{t^{b^{\prime}+n-1} \sum_{k, l=0}^{\infty} \frac{[a, x]_{k+l}(b)_{k}\left(b^{\prime}\right)_{l}}{(c)_{k+l} k ! l !} \frac{z^{k} t^{l}}{(k+l+\alpha)^{r}}\right\} \\
& =\sum_{k=0}^{\infty} \frac{(a)_{k}(b)_{k}}{(c)_{k}} \frac{z^{k}}{k !} \cdot D_{t}^{n}\left\{t^{b^{\prime}+n-1} \sum_{l=0}^{\infty} \frac{[a+k, x]_{l}\left(b^{\prime}\right)_{l}}{(c+k)_{l}} \frac{t^{l}}{l !}\right\} \frac{1}{(k+l+\alpha)^{r}} \\
& =\left(b^{\prime}\right)_{n} t^{b^{\prime}-1} \sum_{k, l=0}^{\infty} \frac{[a, x]_{k+l}(b)_{k}\left(b^{\prime}\right)_{l}}{(c)_{k+l} k ! l !} \frac{z^{k} t^{l}}{(k+l+\alpha)^{r}},
\end{aligned}
$$

which yields the desired result (6.7) asserted by Theorem 7 .

In the same way, we can get the other derivative formulas (6.8) and (6.9) asserted by Theorem 7 .

Corollary 1. The following derivative formulas for the first Appell hypergeometric functions $\Gamma_{1}$ in (3.2) hold true:

$$
\begin{gathered}
D_{t}^{n}\left\{\Gamma_{1}\left[(a, x), b, b^{\prime} ; c ; z, t\right]\right\} \\
=\frac{(a)_{n}\left(b^{\prime}\right)_{n}}{(c)_{n}} \Gamma_{1}\left[(a+n, x), b, b^{\prime}+n ; c+n ; z, t\right], \\
D_{t}^{n}\left\{t^{b^{\prime}+n-1} \Gamma_{1}\left[(a, x), b, b^{\prime} ; c ; z, t\right]\right\} \\
=t^{b^{\prime}-1}\left(b^{\prime}\right)_{n} \Gamma_{1}\left[(a, x), b, b^{\prime}+n ; c ; z, t\right], \\
D_{t}^{n}\left\{t^{c-1} \Gamma_{1}\left[(a, x), b, b^{\prime} ; c ; z t, t\right]\right\} \\
=(-1)^{n} t^{c-n-1}(1-c)_{n} \Gamma_{1}\left[(a, x), b, b^{\prime} ; c-n ; z t, t\right]
\end{gathered}
$$

and

$$
\begin{aligned}
D_{t}^{n}\left\{t^{c-b-1} \Gamma_{1}\left[(a, x), b, b^{\prime} ; c ; z, t\right]\right\}=(-1)^{n} t^{c-b-n-1}(b-c+1)_{n} \\
\cdot \sum_{p=0}^{n}\left(\begin{array}{l}
n \\
p
\end{array}\right) \frac{(a)_{p}\left(b^{\prime}\right)_{p} t^{p}}{(c)_{p}(c-b-n)_{p}} \Gamma_{1}\left[(a+p, x), b, b^{\prime}+p ; c+p ; z, t\right],
\end{aligned}
$$

where $D_{t}^{n}\{f(z, t)\}$ is given by (6.10). 
Proof. Differentiating both sides of the equation (3.2) with respect to $t$ for $n$ times, we can derive the required result (6.12). By using the same operations, we can deduce the desired results (6.13), (6.14) and (6.15).

Remark 2. The special cases of (6.12) to (6.15) when $x=0$ are easily seen to reduce to the known derivative formulas for the Appell hypergeometric function $F_{1}$ (see, for details, [2]).

\section{RECURRENCE RELATIONS}

In this section, we will give the recurrence relation for the extended family of the incomplete Hurwitz-Lerch functions defined by (2.1) and (2.2).

Theorem 8. The following recurrence relation for the function $\varphi_{a, b, b^{\prime} ; c}(z, t, r, \alpha)$ defined by (2.2) holds true:

$$
\begin{aligned}
\varphi_{a, b, b^{\prime} ; c}(z, t, r, \alpha)=\varphi_{a, b, b^{\prime} ; c-1}(z, t, r, \alpha) \\
+\frac{a b z}{c(1-c)} \varphi_{a+1, b+1, b^{\prime} ; c+1}(z, t, r, \alpha+1) \\
+\frac{a b^{\prime} t}{c(1-c)} \varphi_{a+1, b, b^{\prime}+1 ; c+1}(z, t, r, \alpha+1) .
\end{aligned}
$$

Proof. Applying the following well-known contiguous formula for the function ${ }_{0} F_{1}$ given in $[9,20,24]$ :

$$
{ }_{0} F_{1}[-; c-1 ; z]-{ }_{0} F_{1}[-; c ; z]-\frac{z}{c(c-1)}{ }_{0} F_{1}[-; c+1 ; z]=0
$$

to the integral representation in (3.8), we can derive the required result (7.1).

Corollary 2. The following recurrence relations for the function $\varphi_{a, b, b^{\prime} ; c}(z, t, r, \alpha)$ defined by (2.2) hold true:

$$
\begin{aligned}
& \Gamma_{1}\left[(a, x), b+n, b^{\prime} ; c ; z, t\right]=\Gamma_{1}\left[(a, x), b, b^{\prime} ; c ; z, t\right] \\
&+ \frac{a z}{c} \sum_{m=0}^{n} \Gamma_{1}\left[(a+1, x), b+m, b^{\prime} ; c+1 ; z, t\right] . \\
& \Gamma_{1}\left[(a, x), b-n, b^{\prime} ; c ; z, t\right]=\Gamma_{1}\left[(a, x), b, b^{\prime} ; c ; z, t\right] \\
&-\frac{a z}{c} \sum_{m=0}^{n-1} \Gamma_{1}\left[(a+1, x), b-m, b^{\prime} ; c+1 ; z, t\right]
\end{aligned}
$$

and

$$
\begin{aligned}
& \Gamma_{1}\left[(a, x), b, b^{\prime} ; c-n ; z, t\right]=\Gamma_{1}\left[(a, x), b, b^{\prime} ; c ; z, t\right] \\
& +a b z \sum_{m=0}^{n} \frac{\Gamma_{1}\left[(a+1, x), b+1, b^{\prime} ; c+2-m ; z, t\right]}{(c-p)(c-m+1)}
\end{aligned}
$$




$$
+a b^{\prime} t \sum_{m=0}^{n} \frac{\Gamma_{1}\left[(a+1, x), b+1, b^{\prime} ; c+2-m ; z, t\right]}{(c-m)(c-m+1)} .
$$

Proof. Using an obvious identity for $(b+1)_{k}$ in terms of $(b)_{k}$ in the definition (3.2) of the incomplete Appell hypergeometric function $\Gamma_{1}$, we obtain

$$
\begin{aligned}
\Gamma_{1}\left[(a, x), b+1, b^{\prime} ; c ; z, t\right]=\Gamma_{1}\left[(a, x), b, b^{\prime} ; c ; z, t\right] \\
+\frac{a z}{c} \Gamma_{1}\left[(a+1, x), b+1, b^{\prime} ; c+1 ; z, t\right] .
\end{aligned}
$$

Appropriate iteration of this procedure for $n$ times on $\Gamma_{1}$ with the parameter $b+n$, we can get the desired result (7.3).

In the same way as above, we can obtain the recursion relations (7.4) and (7.5).

Theorem 9. The following recurrence relations for the function $\varphi_{a, b, b^{\prime} ; c}(z, t, r, \alpha)$ defined by (2.2) hold true:

$$
\begin{gathered}
\varphi_{a, b+n, b^{\prime} ; c}(z, t, r, \alpha)=\varphi_{a, b, b^{\prime} ; c}(z, t, r, \alpha) \\
+\frac{a z}{c} \sum_{m=0}^{n} \varphi_{a+1, b+m, b^{\prime} ; c+1}(z, t, r, \alpha), \\
\varphi_{a, b-n, b^{\prime} ; c}(z, t, r, \alpha)=\varphi_{a, b, b^{\prime} ; c}(z, t, r, \alpha) \\
-\frac{a z}{c} \sum_{m=0}^{n-1} \varphi_{a+1, b-m, b^{\prime} ; c+1}(z, t, r, \alpha)
\end{gathered}
$$

and

$$
\begin{aligned}
\varphi_{a, b, b^{\prime} ; c-n}(z, t, r, \alpha) & =\varphi_{a, b, b^{\prime} ; c}(z, t, r, \alpha) \\
& +a b z \sum_{m=0}^{n} \frac{\varphi_{a+1, b+1, b^{\prime} ; c+2-m}(z, t, r, \alpha)}{(c-m)(c-m+1)} \\
& +a b^{\prime} t \sum_{m=0}^{n} \frac{\varphi_{a+1, b, b^{\prime}+1 ; c+2-m}(z, t, r, \alpha)}{(c-m)(c-m+1)} .
\end{aligned}
$$

Proof. If we use the equation (7.3) in (3.4), after some simple rearrangements, we can get the desired result (7.7).

In the same way as above, by using the other recursion formulas (7.4) and (7.5) of the incomplete first Appell hypergeometric function $\Gamma_{1}$, we get the equations (7.8) and (7.9).

Corollary 3. The following recurrence relations for the function $\Gamma_{1}\left[(a, x), b, b^{\prime} ; c ; z, t\right]$ defined by (3.2) hold true:

$$
\Gamma_{1}\left[(a, x), b+n, b^{\prime} ; c ; z, t\right]
$$




$$
=\sum_{p=0}^{n}\left(\begin{array}{l}
n \\
p
\end{array}\right) \frac{(a)_{p}}{(c)_{p}} \Gamma_{1}\left[(a+p, x), b+p, b^{\prime} ; c+m ; z, t\right] z^{p}
$$

and

$$
\begin{aligned}
& \Gamma_{1}\left[(a, x), b-n, b^{\prime} ; c ; z, t\right] \\
& \quad=\sum_{p=0}^{n}\left(\begin{array}{l}
n \\
p
\end{array}\right) \frac{(a)_{p}}{(c)_{p}} \Gamma_{1}\left[(a+p, x), b, b^{\prime} ; c+p ; z, t\right](-z)^{p} .
\end{aligned}
$$

Proof. Using the recursion relation (7.6), we can compute the incomplete first Appell hypergeometric function $\Gamma_{1}$ with the parameter $b+2$ as follows:

$$
\begin{aligned}
\Gamma_{1}[(a, x), b+ & \left.2, b^{\prime} ; c ; z, t\right]=\Gamma_{1}\left[(a, x), b, b^{\prime} ; c ; z, t\right] \\
& +\frac{2 a z}{c} \Gamma_{1}\left[(a+1, x), b+1, b^{\prime} ; c+1 ; z, t\right] \\
& +\frac{(a)_{2}}{(c)_{2}} z^{2} \Gamma_{1}\left[(a+2, x), b+2, b^{\prime} ; c+2 ; z, t\right] .
\end{aligned}
$$

Now, by iterating this procedure for $n$ times on $\Gamma_{1}$ with the parameter $b+n$, we obtain the recursion formula (7.10). Similarly, by making use of the same calculations, we can deduce the desired result (7.11).

Theorem 10. The following recurrence relations hold true:

$$
\begin{gathered}
\varphi_{a, b+n, b^{\prime} ; c}(z, t, r, \alpha)=\sum_{m=0}^{n}\left(\begin{array}{l}
n \\
m
\end{array}\right) \frac{(a)_{m}}{(c)_{m}} \\
\cdot \varphi_{a+m, b+m, b^{\prime} ; c+m}(z, t, r, \alpha+m) z^{m}
\end{gathered}
$$

and

$$
\begin{aligned}
& \varphi_{a, b-n, b^{\prime} ; c}(z, t, r, \alpha)=\sum_{m=0}^{n}\left(\begin{array}{c}
n \\
m
\end{array}\right) \frac{(a)_{m}}{(c)_{m}} \\
& \cdot \varphi_{a+m, b+m, b^{\prime} ; c+m}(z, t, r, \alpha+m)(-z)^{m} .
\end{aligned}
$$

Proof. If we use the equation (7.10) in (3.4), after some simple rearrangements, we can derive the desired result (7.12). In precisely the same way, by using the other recursion formula (7.11) for the incomplete first Appell hypergeometric functions $\Gamma_{1}$, we get the equation (7.13).

Remark 3. The special cases of our results (7.3) to (7.5), (7.10) and (7.11) when $x=0$ are easily seen to reduce to the known recursion formulas for the Appell hypergeometric function $F_{1}$ (see [25]). 


\section{REFERENCES}

[1] E. W. Barnes, "The asymptotic expansion of integral functions definied by Taylor's series." Philos. Trans. Roy. Soc. London Ser. A Math. Phys., vol. 206, pp. 249-297, 1906, doi: 10.1098/rsta.1906.0019.

[2] Y. A. Brychkov and N. Saad, "Some formulas for the Appell function $F_{1}\left(a, b, b^{\prime} ; c ; w, z\right)$." Integral Transforms Spec. Funct., vol. 23, pp. 793-802, 2012, doi: 10.1080/10652469.2011.636651.

[3] A. Çetinkaya, "The incomplete second Appell hypergeometric functions." Appl. Math. Comput., vol. 219, pp. 8332-8337, 2013, doi: 10.1016/j.amc.2012.11.050.

[4] M. A. Chaudhry and S. M. Zubair, On a Class of Incomplete Gamma Functions with Applications. Boca Raton, London, New York and Washington, D. C.: Chapman and Hall (CRC Press Company), 2001. doi: 10.1201/9781420036046.

[5] J. Choi, D. S. Jang, and H. M. Srivastava, "A generalization of the Hurwitz-Lerch zeta function." Integral Transforms Spec. Funct., vol. 19, pp. 65-79, 2008, doi: 10.1080/10652460701528909.

[6] J. Choi and R. K. Parmar, "An Extension of the Generalized Hurwitz-Lerch Zeta Function of Two Variables.” Filomat, vol. 31, pp. 91-96, 2017, doi: 10.2298/FIL1701091C.

[7] J. Choi, R. K. Parmar, and P. Chopra, "The incomplete Lauricella and first Appell functions and associated properties." Honam Math. J., vol. 36, pp. 531-542, 2014, doi: 10.5831/HMJ.2014.36.3.531.

[8] O. A. Daman and M. A. Pathan, "A further generalisation of the Hurwitz zeta function." Math. Sci. Res. J., vol. 16, pp. 251-259, 2012, doi: 10.1016/j.amc.2012.11.050.

[9] A. Erdélyi, W. Magnus, F. Oberhettinger, and F. G. Tricomi, Higher Transcendental Functions. Vol. I. New York, Toronto, London and Hong Kong: McGraw-Hill Book Company, 1953.

[10] M. Garg, K. Jain, and S. L. Kalla, "A further study of general Hurwitz-Lerch zeta function." Algebras Groups Geom., vol. 25, pp. 311-319, 2008.

[11] S. P. Goyal and R. K. Laddha, "On the generalized Riemann zeta functions and the generalized Lambert transform.” Ganita Sandesh, vol. 11, pp. 99-108, 1997.

[12] P. L. Gupta, R. C. Gupta, S.-H. Ong, and H. M. Srivastava, "A class of Hurwitz-Lerch zeta distributions and their applications in reliability." Appl. Math. Comput., vol. 196, pp. 521-531, 2008, doi: 10.1016/j.amc.2007.06.012.

[13] S.-D. Lin and H. M. Srivastava, "Some families of the Hurwitz-Lerch zeta functions and associated fractional derivative and other integral representations." Appl. Math. Comput., vol. 154, pp. 725 733, 2004, doi: 10.1016/S0096-3003(03)00746-X.

[14] S.-D. Lin, H. M. Srivastava, and P.-Y. Wang, "Some expansion formulas for a class of generalized Hurwitz-Lerch zeta functions.” Integral Transforms Spec. Funct., vol. 17, pp. 817-827, 2006, doi: 10.1080/10652460600926923.

[15] H. M. Srivastava, "A new family of the $\lambda$-generalized Hurwitz-Lerch Zeta functions with applications.” Appl. Math. Inform. Sci., vol. 8, pp. 1485-1500, 2014, doi: 10.12785/amis/080402.

[16] H. M. Srivastava, M. A. Chaudhry, and R. P. Agarwal, "The incomplete Pochhammer symbols and their applications to hypergeometric and related functions." Integral Transforms Spec. Funct., vol. 23, pp. 659-683, 2012, doi: 10.1080/10652469.2011.623350.

[17] H. M. Srivastava and J. Choi, Series Associated with the Zeta and Related Functions. Dordrecht, Boston and London: Kluwer Acedemic Publishers, 2001.

[18] H. M. Srivastava and J. Choi, Zeta and q-Zeta Functions and Associated Series and Integrals. Amsterdam, London and New York: Elsevier Science Publishers, 2012.

[19] H. M. Srivastava, D. Jankov, T. K. Pogány, and R. K. Saxena, "Two-sided inequalities for the extended Hurwitz-Lerch zeta function.” Comput. Math. Appl., vol. 62, pp. 516-522, 2011, doi: 10.1016/j.camwa.2011.05.035. 
[20] H. M. Srivastava and P. W. Karlsson, Multiple Gaussian hypergeometric Series. New York, Chichester, Brisbane and Toronto: Halsted Press (Ellis Horwood Limited), John Wiley and Sons, 1985.

[21] H. M. Srivastava, M.-J. Luo, and R. Raina, "New results involving a class of generalized Hurwitzlerch zeta functions and their applications." Turkish J. Anal. Number Theory, vol. 1, pp. 26-35, 2013, doi: 10.12691/tjant-1-1-7.

[22] H. M. Srivastava and H. L. Manocha, A Treatise on Generating Functions. New York, Chichester, Brisbane and Toronto: Halsted Press (Ellis Horwood Limited), John Wiley and Sons, 1984.

[23] H. M. Srivastava, R. K. Saxena, and R. K. Parmar, "Some families of the incomplete $H$ functions and the incomplete $\bar{H}$-functions and associated integral transforms and operators of fractional calculus with applications." Russian J. Math. Phys., vol. 25, pp. 116-138, 2018, doi: 10.1134/S1061920818010119.

[24] H. M. Srivastava, R. K. Saxena, T. K. Pogány, and R. Saxena, "Integral and computational representations of the extended Hurwitz-Lerch zeta function." Integral Transforms Spec. Funct., vol. 22, pp. 487-506, 2011, doi: 10.1080/10652469.2010.530128.

[25] X.-X. Wang, "Recursion formulas for Appell functions." Integral Transforms Spec. Funct., vol. 23, pp. 421-433, 2012, doi: 10.1142/S1793557115500825.

\section{Authors' addresses}

\section{H. M. Srivastava}

Department of Mathematics and Statistics, University of Victoria, Victoria, British Columbia V8W 3R4, Canada and Department of Medical Research, China Medical University Hospital, China Medical University, Taichung 40402, Taiwan, Republic of China and Department of Mathematics and Informatics, Azerbaijan University, 71 Jeyhun Hajibeyli Street, AZ1007 Baku, Azerbaijan

E-mail address: harimsriemath.uvic.ca

\section{Recep Sahin}

Department of Mathematics, Faculty of Arts and Sciences, Kırıkkale University, TR-71450 Kırıkkale, Turkey

E-mail address: recepsahin@kku.edu.tr

\section{Ŏguz Yağcı} Turkey

Department of Mathematics, Faculty of Arts and Sciences, Kırıkale University, TR-71450 Kırıkkale, E-mail address: oguzyagci26@gmail.com 